La Revue

des Droits

de l'Homme

\section{La Revue des droits de l'homme}

Revue du Centre de recherches et d'études sur les droits fondamentaux

Actualités Droits-Libertés | 2015

\title{
L'orientation sexuelle comme motif de persécution doit être appréciée dans la dignité
}

Droit d'asile (Directive 2004/83/CE \& Directive 2005/85/CE)

\section{Marion Tissier-Raffin}

\section{OpenEdition}

Journals

Édition électronique

URL : https://journals.openedition.org/revdh/1048

DOI : $10.4000 /$ revdh. 1048

ISSN : 2264-119X

Éditeur

Centre de recherches et d'études sur les droits fondamentaux

Référence électronique

Marion Tissier-Raffin, «L'orientation sexuelle comme motif de persécution doit être appréciée dans la dignité », La Revue des droits de l'homme [En ligne], Actualités Droits-Libertés, mis en ligne le 15 janvier 2015, consulté le 24 janvier 2022. URL : http://journals.openedition.org/revdh/1048 ; DOI : https:// doi.org/10.4000/revdh. 1048

Ce document a été généré automatiquement le 24 janvier 2022.

Tous droits réservés 


\section{L'orientation sexuelle comme motif de persécution doit être appréciée dans la dignité}

Droit d'asile (Directive 2004/83/CE \& Directive 2005/85/CE)

Marion Tissier-Raffin

1 C'est une nouvelle fois à l'initiative du Conseil d'Etat des Pays-Bas que la CJUE a donc été amenée à se prononcer sur la conformité au droit de l'Union européenne de l'appréciation nationale de la qualité de réfugié des personnes craignant d'être persécutées en raison de leur orientation sexuelle. Dans un arrêt X., Y., \& Z. c/ Minister voor Immigratie en Asiel, du 7 novembre 2013'1 la CJUE s'était prononcée sur l'appréciation des conditions de fond auxquelles ces demandeurs d'asile doivent satisfaire pour se voir reconnaître la qualité de réfugié. Dans l'arrêt ici étudié, elle doit examiner la légalité des preuves exigées ou volontairement déposées par ces mêmes requérants pour justifier leur orientation sexuelle.

2 En droit européen de l'asile, cette décision intervient au terme d'un processus d'assouplissement de l'interprétation des critères de reconnaissance de la qualité de réfugié pour les demandeurs d'asile invoquant des craintes de persécution en raison de leur orientation sexuelle, conduisant les autorités nationales à se focaliser de plus en plus sur la crédibilité de l'orientation sexuelle alléguée ${ }^{2}$. Or, l'appréciation de cette crédibilité a pu mener à des dérives, récemment dénoncées publiquement dans plusieurs pays européens ${ }^{3}$, tant les interrogatoires menés ou les preuves exigées ont pu paraître à juste titre abusifs, c'est-à-dire violant la dignité et la vie privée des demandeurs d'asile. C'est pourquoi le Conseil d'Etat néerlandais demandait à la CJUE si les Etats-membres doivent se contenter d'accepter l'orientation sexuelle alléguée des demandeurs d'asile comme le soutenaient leurs avocats ou s'ils peuvent, comme pour tout autre motif de persécution, examiner la crédibilité de celle-ci ${ }^{4}$, cette deuxième alternative imposant de déterminer les limites à respecter, notamment la collecte des éléments de preuves, pour garantir le respect de la dignité des demandeurs d'asile concernés ${ }^{5}$. 
Ces questions, qui soulèvent des interrogations complexes d'ordre conceptuel portant sur la définition de la sexualité, mettent autant en jeu les droits fondamentaux des demandeurs d'asile que l'efficacité du régime d'asile européen commun qui ne peut tolérer des divergences portant sur les modes de preuves exigées. Sans remettre en cause le droit des Etats d'examiner le bien-fondé des demandes d'asile, y compris l'orientation sexuelle alléguée par les requérants, la CJUE a néanmoins fermement fixé des limites à ces vérifications pour éviter toute dérive de nature à violer leurs droits fondamentaux. Elle s'est appuyée sur la Directive 2004/83/CE pour rappeler aux Etats les principes généraux devant guidés l'appréciation de la crédibilité de toute demande d'asile et sur la Charte des droits fondamentaux pour fixer des limites infranchissables dans l'évaluation de la crédibilité de l'orientation sexuelle. Toutefois, et on peut le regretter, elle n'a pas défendu explicitement une conception non superficielle et non stéréotypée de l'orientation sexuelle comme point de départ de toute appréciation de son caractère plausible, ni fait preuve d'une pleine compréhension des motifs de persécution permettant de justifier la reconnaissance de la qualité de réfugié.

4 Parce que l'appréciation de la crédibilité de l'orientation sexuelle des demandeurs d'asile est devenue l'enjeu central de la détermination de la qualité de réfugié $\left(1^{\circ}\right)$, il est ainsi devenu indispensable que la CJUE établisse les principes et les limites infranchissables que les autorités nationales doivent respecter lorsqu'elles apprécient la crédibilité de l'orientation sexuelle alléguée par les demandeurs d'asile $\left(2^{\circ}\right)$.

\section{$1 \%$ - L'appréciation de la crédibilité de l'orientation sexuelle alléguée : nouvel enjeu central de l'appréciation de la qualité de réfugié}

5 Si les évolutions récentes portant sur l'interprétation des critères d'éligibilité au statut de réfugié ont conduit à l'élargissement des bénéficiaires de la qualité de réfugié, elles ont aussi conduit les autorités nationales à se focaliser sur l'appréciation de la crédibilité de l'orientation sexuelle alléguée par les requérants car elle est devenue le dernier et principal motif permettant de refuser ce statut. Or, dans un climat de suspicion généralisée à l'égard de toute demande d'asile, l'appréciation de la crédibilité $\mathrm{du}$ récit des demandeurs d'asile suscitent de nombreuses critiques, particulièrement quand elle porte sur le caractère plausible de l'orientation sexuelle alléguée, en ce qu'elle a abouti à des dérives stupéfiantes publiquement dénoncées dans plusieurs pays européens.

6 Depuis une vingtaine d'années, l'interprétation des critères de l'article 1A définissant la qualité de réfugié, repris à l'article 2 d) de la Directive 2011/95/UE, n'a cessé d'évoluer dans la plupart des Etats européens. Les obstacles liés à l'interprétation de ces critères pouvant encore justifier le refus de la qualité de réfugié aux personnes fuyant des violences liées à leur orientation sexuelle ont donc été levés. Ces évolutions ont été consacrées en Europe par la CJUE en 2013 dans un arrêt X, $Y$ et $Z$ c/ Minister voor Immigratie en Asiel' ${ }^{6}$. Même si plusieurs critiques ont été émises à juste titre à l'encontre de cet arrêt ${ }^{7}$, celui-ci reste d'une importance capitale car il a consacré en droit européen de l'asile la protection dont doivent bénéficier les individus victimes de persécution en raison de leur orientation sexuelle. 
7 Ni la Directive «Qualification» (Directive 2004/83/CE - Directive 2011/95/UE (refonte)), ni la Directive relative aux procédures d'asile (Directive 2005/85/CE - Directive 2013/32/UE (refonte)), ni même la Convention de Genève ne prévoient de dispositions particulières quant à la manière dont la crédibilité d'un demandeur qui sollicite le statut de réfugié sur le fondement de son appartenance à un certain groupe social en raison de son orientation sexuelle doit être évaluée. Dès lors, cette appréciation doit se fonder, comme tout autre demande et comme la Cour l'a rappelé 8 , sur l'article 4 de la Directive 2004/83, contenu au chapitre II de celle-ci, portant sur « l'évaluation des faits et circonstances». Or, si l'appréciation de la crédibilité des données factuelles des demandeurs d'asile répond à un encadrement flou emportant de nombreuses critiques (A), celle portant plus spécifiquement sur la vérification d'une orientation sexuelle alléguée pose de sérieuses difficultés conceptuelles et juridiques spécifiques et peut rapidement aboutir à des dérives choquantes dans la pratique (B).

\section{A - Les règles encadrant « les conditions d'évaluation des faits et des circonstances » d'une demande de protection internationale.}

8 Comme tout autre motif invoqué à l'appui d'une demande de protection internationale, l'évaluation de son bien-fondé est réglementée par l'article 4 de la Directive 2011/95/ UE. Cette disposition précise tout d'abord que les Etats-membres sont tenus d'évaluer toutes les demandes de protection internationale. Et selon l'arrêt M.M de la CJUE du 22 novembre $2012^{9}$, cette évaluation "se déroule en deux étapes distinctes ${ }^{10}$. La première étape consiste à analyser le caractère plausible des éléments factuels du récit exposé par les requérants et la seconde à confronter ce récit aux critères d'éligibilité au statut de réfugié. L'appréciation de la crédibilité du récit, et donc de l'orientation sexuelle du requérant, relève ainsi de la première étape.

9 Dans le cadre de celle-ci, il revient alors au demandeur la charge de présenter tous les éléments nécessaires pour étayer sa demande. L'article $4 \$ 1$ impose également une obligation positive à la charge des Etats de coopérer activement avec le demandeur pour permettre la réunion de l'ensemble des éléments de nature à étayer sa demande ${ }^{11}$. En effet, il n'est pas rare que les Etats membres, tenus de collecter des informations précises et actualisées sur la situation générale existant dans les pays d'origine des demandeurs d'asile (article 8 \$2b de la Directive 2005/85/CE du $1^{\text {er }}$ décembre 2005 article $10 \$ 3$ de la Directive 2013/32/UE (refonte)), soient mieux placés que ces derniers pour avoir accès à certains types de documents ${ }^{12}$. De plus, comme le précise l'article 4 $\$ 5$ de la Directive 2004/83/CE (article $4 \$ 5$ de la Directive 2011/95/UE (refonte)), les requérants sont rarement en mesure d'apporter des preuves documentaires à l'appui de leur récit individuel. C'est pourquoi cette disposition reconnaît qu'en l'absence de telles preuves, les déclarations du requérant ne nécessitent pas de confirmation lorsque celui-ci s'est "réellement efforcé d'étayer sa demande» (a), l'a "présentée dès que possible» (d), «a présenté des explications satisfaisantes quant à l'absence d'éléments probants» (b), et «lorsque ses déclarations sont jugées cohérentes et plausibles » (c), et de manière générale, "la crédibilité de son récit peut être établie »(e). Dans la réalité, en l'absence de preuves documentaires, l'appréciation des faits et circonstances factuels repose donc très souvent sur l'appréciation de la crédibilité du récit, c'est-à-dire le caractère vraisemblable et plausible des motifs exposés par le demandeur pour expliquer pourquoi il a fui son pays et pourquoi il craint d'y retourner. 
Or, pour mener l'évaluation de la crédibilité du récit, les autorités nationales ne sont pas liées par des règles techniques ou légales. C'est ainsi que l'intime conviction des personnes en charge de reconnaître la qualité de réfugié occupe une place primordiale, alors qu'elle repose sur la capacité du requérant à convaincre celles-ci de la véracité plausible de son récit $^{13}$. Parce qu'emporter l'intime conviction des autorités compétentes est à la fois déterminant et difficile, on assiste à une véritable course aux preuves objectives, tels que les certificats médicaux, susceptibles d'objectiver et de crédibiliser un récit imprécis ou présentant des incohérences temporelles ou internes ${ }^{14}$. C'est donc dans ce cadre légal imprécis et ce contexte général de suspicion que se pose la question de l'appréciation plus spécifique de la crédibilité d'une orientation sexuelle alléguée.

\section{B - L'appréciation de l'orientation sexuelle alléguée : enjeux spécifiques et dérives étatiques}

11 Si la vérification des données factuelles exposées par les demandeurs d'asile est à la fois légitime pour identifier les réfugiés statutaires et une obligation communautaire à la charge des Etats-membres, peut-on vérifier la crédibilité d'une orientation sexuelle comme tout autre motif de persécution ? Si oui, la méthode retenue peut-elle être identique aux autres motifs de persécution? Pour répondre à ces questions, et comprendre les enjeux qu'elles soulèvent, il est nécessaire de définir conceptuellement et légalement l'orientation sexuelle (1), avant d'exposer les dérives auxquelles cette évaluation a pu aboutir dans certains Etats européens (2).

\section{1 - Le droit à l'auto-détermination de son orientation sexuelle}

12 Depuis $1^{\text {er }}$ mai 1990 , l'homosexualité n'est plus considérée par l'OMS comme une maladie mentale ${ }^{15}$. Selon les principes de Yogjakarta ${ }^{16}$, qui reflètent l'état actuel du droit international des droits humains en rapport avec les enjeux relatifs à l'orientation sexuelle et l'identité de genre, l'orientation sexuelle fait référence à "la capacité de chacun à ressentir une profonde attirance émotionnelle, affective et sexuelle envers des individus du sexe opposé, de même sexe ou de plus d'un sexe, et d'entretenir des relations intimes et sexuelles avec ces individus». Elle se différencie de l'identité de genre qui renvoie «à l'expérience intime et sexuelle du sexe faite par chacun, qu'elle corresponde ou non au sexe assigné à la naissance, y compris une conscience personnelle du corps (...) et d'autres expressions du sexe, y compris l'habillement, le discours et les manières de se conduire ».

13 Mais, comme le rappelle l'UNHCR dans ses Principes Directeurs $n^{\circ} 9^{17}$, les demandes fondées sur une différence d'orientation sexuelle contiennent un élément lié au genre. En effet, dans de nombreux cas, les personnes homosexuelles refusent de se conformer aux rôles sociaux ou culturels prédéfinis ou aux comportements attribués à leur sexe biologique. Comme l'ont reconnu l'Avocate Générale et l'ensemble des parties, l'orientation sexuelle et l'identité de genre sont donc des sujets extrêmement complexes et liés ${ }^{18}$. Si l'orientation sexuelle d'une personne peut être révélée par son comportement sexuel, elle peut aussi l'être par l'apparence extérieure, par l'habillement, la manière dont la personne vit en société ou exprime son identité, etc. Par ailleurs, si l'orientation sexuelle est pour la plupart des personnes déterminée à un âge précoce, pour d'autres, elle peut continuer à évoluer tout au long de la vie; son 
expression peut varier avec l'âge de même qu'en fonction d'autres déterminants personnels, familiaux, sociaux ou culturels ${ }^{19}$.

14 L'orientation sexuelle est donc un concept très vaste qui touche au plus profond de l'identité personnelle des individus. C'est la raison pour laquelle, en droit, l'orientation sexuelle est rattachée au droit fondamental des individus au respect de leur vie privée $^{20}$. Les expériences sont tellement diverses et variées qu'il est quasi impossible de mener des comparaisons, de s'appuyer sur des normes exposant la manière dont sont supposées se comporter les personnes concernées, sauf à tomber dans une compréhension superficielle du vécu de ces personnes, ou à s'appuyer sur des hypothèses erronées, culturellement inappropriées ou stéréotypées. C'est pourquoi l'UNHCR a reconnu que l'orientation sexuelle devait relever de l'auto-identification des personnes concernées ${ }^{21}$. Si la Cour européenne des droits de l'homme ne s'est pas à ce jour prononcée explicitement sur la reconnaissance d'un droit à l'auto-détermination, elle a toutefois jugé que « la notion d'autonomie personnelle reflète un principe important qui sous-tend l'interprétation des garanties de l'article 8 de la $C_{E D H}{ }^{22} »$. Les particuliers sont donc en droit de définir leur propre orientation sexuelle.

15 Si l'orientation sexuelle d'une personne, qui relève du droit au respect de sa vie privée, est indissolublement liée à son identité personnelle et si complexe à examiner que seul l'individu lui-même est finalement à même de la définir, se pose donc la question de savoir comment une autorité extérieure peut vérifier la crédibilité d'une orientation sexuelle alléguée. Tel est le cœur de la problématique soulevée par le Conseil d'Etat néerlandais. Selon l'avocat des parties, qui reprend d'ailleurs l'argumentation défendue par les associations de défense des personnes LGBT, les autorités nationales ne sont pas compétentes pour substituer leur appréciation à la déclaration du demandeur quant à son orientation sexuelle puisque qu'il n'existe pas de caractéristiques générales propres à l'homosexualité ni de consensus quant aux facteurs qui influent sur une telle orientation sexuelle ${ }^{23}$. Autrement dit, ces autorités devraient considérer la prétendue orientation sexuelle d'une requérant comme un fait établi sur la base de ses seules déclarations ${ }^{24}$. Cette position n'est cependant pas celle défendue par de nombreux Etats européens. Selon ces derniers, les autorités nationales sont compétentes pour examiner le caractère crédible d'une orientation sexuelle alléguée, comme elles le sont pour apprécier la crédibilité de tout autre motif de persécution ${ }^{25}$. Mais dans la réalité, non seulement ces derniers ne procèdent pas à l'évaluation de la crédibilité d'une orientation sexuelle alléguée de manière très uniforme, mais certaines des pratiques mises en œuvre se sont traduites par la mise en place de questionnaires et/ou d'examens choquants ${ }^{26}$.

\section{2 - Les dérives étatiques.}

16 Comme l'explique l'avocat anglais Colin Yeo, la culture de la suspicion est telle que tous les agents pensent avoir à faire à de faux demandeurs d'asile. Or, quand il s'agit d'apprécier une donné factuelle aussi complexe que l'orientation sexuelle, cette logique peut aboutir à des pratiques intrusives ${ }^{27}$. Voici quelques exemples de pratiques dites «choquantes» qui ont récemment provoqué l'indignation des associations de défense des personnes LGBT ou réfugiés, ou dont la révélation publique a pu provoquer l'indignation de l'ensemble des citoyens. 2014, The Observer publiait un article en Grande-Bretagne reproduisant des 
extraits d'un entretien mené dans le cadre de la détermination d'une demande de protection internationale dans laquelle le demandeur d'asile a été soumis pendant près de 5 heures à des questions jugées "choquantes et humiliantes" portant sur des détails de sa vie sexuelle et révélant une compréhension stéréotypée de l'orientation sexuelle ${ }^{28}$. Lui ont ainsi été posées des questions telles que : «Did you put your penis into x's backside? When $x$ was penetrating you? Did you have an erection? Did $x$ ejaculate inside you? What is it about men's backside that attracts you? What is it about the way men walk that turns you on?». L'indignation provoquée par ces révélations a été telle qu'une étude indépendante a été mandatée par le Ministère de l'Intérieur ${ }^{29}$. Publiée en octobre 2014, cette étude montre que $10 \%$ des questions posées sont encore aujourd'hui intrusives et abusives. Elle révèle aussi que, dans le cadre des procédures accélérées, les agents acceptent des documents vidéo ou photographiques exposant la vie sexuelle des requérants comme mode de preuve attestant de la véracité de leur orientation sexuelle. Selon le rapport "Fleeing Homophobia" mené par Sabine JANSEN et Thomas SPIJKERBOER ${ }^{30}$, on retrouverait de telles questions dans plusieurs Etats européens, dont l'Allemagne et la Belgique.

Outre ces questionnaires ahurissants, d'autres pratiques stupéfiantes étaient encore très récemment appliquées en République Tchèque et en Slovaquie. Il s'agit de la mise en œuvre en 2008 des tests phallométriques (ou pléthysmographie vaginale pour les femmes). Ces derniers ont pour but de mesurer le changement opéré dans la circonférence et le volume du pénis durant la présentation de stimulis audiovisuels. Outre que ces "pseudo tests médicaux» sont très critiqués dans le monde médical, ils exposaient surtout les personnes à des examens intrusifs pour les requérants (supports souvent de caractère pornographiques). Cette méthode a provoqué une telle indignation de UE, de l'UNHCR et des associations de défense des personnes LGBT, qu'elle a finalement été abandonnée en $2009^{31}$.

Il n'est pas non plus rare que les autorités nationales se fondent sur une compréhension stéréotypée de l'orientation sexuelle lorsqu'ils questionnent les requérants homosexuels. Par exemple, il est fréquent que l'absence de connaissance de «la culture homosexuelle» (clubs, sites internet, organisation de défense des personnes LGBT, littérature, magasines) soit interprétée comme une remise en cause de la crédibilité de l'orientation sexuelle alléguée par le requérant. Toujours selon le rapport Fleeing Homophobia, on retrouve ce type de raisonnement dans de nombreux pays, comme la France, la Belgique, l'Irlande et les Pays-Bas ${ }^{32}$.

Enfin, il apparaît que de plus en plus de requérants déposent volontairement des photos ou des enregistrements vidéo mettant en scène leur rapport sexuel pour appuyer leur demande d'asile, alors même que les autorités nationales n'exigent aucunement de tels types de preuve. Cette pratique s'inscrit évidemment dans un contexte global voulant que tout demandeur d'asile doit déposer le maximum de preuves objectives susceptibles de crédibiliser un récit systématiquement considéré comme suspect ${ }^{33}$.

21 On comprend donc pourquoi l'intervention de la CJUE était nécessaire pour plusieurs raisons. D'abord pour uniformiser les pratiques relatives au mode de preuves exigées afin de ne pas mettre en péril l'efficacité recherché du système européen d'asile. Ensuite et surtout pour apprécier la légalité de l'ensemble de ces pratiques intrusives et choquantes au regard du droit européen de l'asile et des droits fondamentaux des demandeurs d'asile. 


\section{$2 \%$ - L'appréciation de la crédibilité de l'orientation sexuelle alléguée : principes généraux et limites spécifiques infranchissables}

motifs autres que la persécution en raison de son homosexualité avant d'invoquer celle-ci comme motif de persécution dans le cadre d'une seconde demande d'asile. C a alors soutenu qu'il n'avait pas pu "reconnaitre son attirance homosexuelle avant d'avoir quitté son pays d'origine $»^{38}$ et il a remis aux autorités un enregistrement vidéo d'actes intimes avec une personne du même sexe à l'appui de sa demande. Là encore, sa demande d'asile a été rejetée pour défaut de crédibilité. Les autorités ont considéré qu'il aurait dû invoquer son orientation sexuelle lors de sa première demande d'asile et que le seul fait qu'il ait déposé un film le montrant dans un rapport sexuel avec un homme n'établissait pas qu'il était homosexuel. Enfin, elles ont jugé que le requérant n'avait pas suffisamment explicité «comment il avait pris conscience de son orientation sexuelle », et qu'il «ne pouvait répondre à des questions portant notamment sur l'identification d'organisations de défense des droits des homosexuels aux Pays-Bas $»^{39}$.

Concernant ces trois affaires, la juridiction de renvoi demandait donc à la CJUE de se prononcer sur la légalité des pratiques observées. Plus précisément, elle demandait à la Cour s'il existait en droit de l'UE des limitations imposées à la manière dont est apprécié le caractère crédible d'une orientation sexuelle prétendue et si ces limitations sont différentes de celles valant pour l'appréciation du caractère crédible d'autres motifs de persécution. Pour répondre à cette question, la CJUE s'est alors fondée sur le droit européen de l'asile et la Charte des droits fondamentaux pour en tirer des 
principes généraux d'encadrement $(\mathrm{A})$ et établir de manière ferme quatre limites infranchissables (B).

\section{A - Principe d'application générale : une appréciation individuelle dans le respect des droits fondamentaux des demandeurs d'asile}

S'appuyant sur l'article 4 de la Directive 2004/83/CE (Article 4 de la Directive 2011/95/ UE (refonte)) et la Charte des droits fondamentaux, la CJUE a rappelé trois grands principes encadrant l'évaluation du caractère plausible de l'orientation sexuelle d'un requérant, dont deux ont une portée qui va bien au-delà du seul cas d'espèce. Il s'agit du droit des Etats d'évaluer la crédibilité d'une orientation sexuelle alléguée (1), de l'obligation de mener une évaluation individuelle (2) et de l'obligation de respecter les droits fondamentaux des requérants (3).

\section{1 - Le droit des Etats d'évaluer la crédibilité d'une orientation sexuelle alléguée}

La demande des avocats des demandeurs d'asile de ne pas procéder à l'évaluation de l'orientation sexuelle des requérants et de se fonder sur leur seule déclaration n'a pas connu de réponse favorable de la part de la CJUE. Cette dernière a au contraire reconnu le droit des Etats d'examiner cet élément de leur demande. Toutefois, elle a précisé, comme le recommandait d'ailleurs l'UNHCR ${ }^{40}$, que les déclarations soient le «point de départ » de cette évaluation ${ }^{41}$. La Cour reconnaît ainsi explicitement qu' " il appartient au demandeur d'asile d'identifier» son orientation sexuelle car elle "constitue un élément relevant de sa sphère personnelle ». Ce premier principe s'appuie donc sur une conciliation entre les obligations à la charge des Etats, au nom du droit d'asile européen de procéder à l'évaluation de toutes les demandes d'asile et le droit fondamental des personnes de procéder à l'identification de leur propre orientation sexuelle.

Malgré la reconnaissance de l'auto-identification comme point de départ de toute évaluation de l'orientation sexuelle, on peut regretter la timidité avec laquelle la Cour affirme ce principe. Celle-ci aurait pu exposer ce premier principe d'encadrement plus fermement comme l'a fait l'Avocate Générale, pour qui les déclarations des requérants doivent être "nécessairement » et «toujours » le point de départ de l'évaluation de la crédibilité d'une orientation sexuelle invoquée comme motif de persécution ${ }^{42}$.

\section{2 - L'obligation des Etats de procéder à un examen individuel}

La Cour a ensuite rappelé que, comme l'exige l'article $4 \$ 3$ c) de la Directive 2004/83/CE (Article $4 \$ 3$ de la Directive 2011/95/UE (refonte)), toute évaluation des faits et circonstances évoqués par les demandeurs d'asile doit faire l'objet « d'une appréciation individuelle ${ }^{43}$. Cela signifie que les autorités en charge de cette évaluation doivent tenir compte " du statut individuel ainsi que la situation personnelle du demandeur, y compris des facteurs tels que son passé, son sexe, son âge ". En effet, la manière dont une personne exprime son orientation sexuelle peut varier en fonction de nombreux facteurs. Lorsque les autorités évaluent le caractère crédible d'une orientation sexuelle alléguée, elles ne peuvent donc pas se fonder sur une conception stéréotypée de celle-ci sauf à violer la règle de l'individualité de l'évaluation. Le rappel de ce principe était primordial pour les espèces en cause car il rejette toute compréhension superficielle et stéréotypée de l'homosexualité. 
31 Cègle a aussi une portée plus générale: il n'est pas légal d'attendre des demandeurs d'asile qu'ils apportent des réponses préconçues aux questions qui leur sont posées, sauf à ne pas respecter le fait que les craintes à l'origine des persécutions invoquées doivent être individuelles selon l'article $1 \mathrm{~A}$ de la Convention de Genève. Or, comme l'a rappelé la CJUE, la Convention de Genève "constitue la pierre angulaire $d u$ régime juridique international de protection des réfugiés » et la Directive 2004/84/CE a été adoptée « pour aider les autorités compétentes des Etats membres à appliquer cette convention (...) $\rrbracket^{44}$. La Cour s'inscrit donc ici dans la continuité des principes généraux encadrant l'interprétation de l'article $1 \mathrm{~A}$ de la Convention de Genève qui définit la qualité de réfugié ${ }^{45}$.

\section{3 - L'obligation des Etats de respecter les droits fondamentaux des demandeurs d'asile}

principe d'encadrement dégagé par la CJUE est de la plus haute importance. Il se fonde sur le fait que la Directive 2004/83/CE (considérant 16 de la Directive 2011/95/UE (refonte)), et notamment l'interprétation et l'application de son article 4, doit respecter les droits reconnus dans la Charte ${ }^{46}$. Ce rappel de la CJUE était absolument nécessaire pour les cas d'espèce et sa portée va au-delà des demandes d'asile invoquant l'orientation sexuelle comme motif de persécution. Toutes les demandes d'asile, lorsqu'elles font l'objet d'un examen portant sur l'évaluation des faits et circonstances à l'origine du refus des personnes de retourner dans leurs pays parce qu'elles craignent d'y être persécutées, doivent faire l'objet d'un examen qui respectent les droits fondamentaux proclamés dans la Charte. Cette affirmation est certes une évidence pour les juristes, mais pour les requérants invoquant des persécutions en raison de leur orientation sexuelle, elle emporte des conséquences très concrètes. Elle fonde en droit la fixation de limites infranchissables très claires dans la manière de procéder à l'examen de l'orientation sexuelle des demandeurs d'asile. Car cet examen doit notamment être mené dans le respect de la dignité humaine (article $1^{\text {er }}$ de la Charte), et du droit à la vie privée et familiale (article 7 de la Charte) ${ }^{47}$.

\section{B - L'appréciation de la crédibilité de l'orientation sexuelle alléguée : les pratiques interdites}

Sur le fondement de ces trois principes d'encadrement, la CJUE a strictement interdit quatre pratiques pour évaluer la crédibilité d'une orientation sexuelle alléguée par les demandeurs d'asile. Ces quatre limites reprennent les observations de l'UNHCR ${ }^{48}$. Il s'agit des interrogatoires portant sur les détails de la vie sexuelle des requérants (1), des preuves se basant sur des tests médicaux, des vidéo ou des photographies (2), de la possibilité de rejeter une demande d'asile pour défaut de crédibilité quand le requérant a déclaré tardivement son orientation sexuelle (3) et de la possibilité de rejeter une telle demande sur le seul fondement de réponses "incorrectes" apportées à des questionnaires élaborés sur la base d'une conception stéréotypées de l'orientation sexuelle (4). 


\section{1 - L'interdiction des interrogatoires portant sur des détails de la vie sexuelle des requérants.}

La CJUE a d'abord explicitement condamnée " les interrogatoires concernant les détails des pratiques sexuelles des demandeurs d'asile" au motif que ces derniers étaient " contraires aux droits fondamentaux garantis par la Charte, et notamment, au droit au respect de la vie privée et familiale (...) $\aleph^{49}$. C'est le caractère intrusif des questions posées, parce qu'elles touchent au plus intime, qui est ici reconnu. Cette première limitation fait donc droit à la demande des associations de défense des personnes LGBT et aux recommandations émises par l'UNHCR. Elle vient désavouer un certain nombre de pratiques qui perdurent encore dans plusieurs pays européens. Elle témoigne surtout d'une conception large de l'orientation sexuelle qui ne doit pas se limiter pas à l'acte ou au comportement sexuel de l'individu.

\section{2 - L'interdiction des preuves se basant sur des tests médicaux, des vidéo ou des photographies}

La CJUE a aussi condamné la possibilité "pour les autorités nationales d'accepter l'accomplissement d'actes homosexuels, leur soumission à d'éventuels "tests" en vue d'établir leur homosexualité ou encore la production par les demandeurs de preuves telles que des enregistrements vidéo de leurs actes intimes" au motif que ces pratiques sont "de nature à porter atteinte à la dignité humaine $"^{50}$. Par cette affirmation, la Cour vise d'abord tous les tests pseudo-médicaux, ce qui comprend le test de phallométrie. Toutefois, en ne le nommant pas explicitement et en employant un vocabulaire volontairement large et neutre, la CJUE vise tous les tests possibles et à venir qui pourraient se prévaloir de leur caractère prétendument médical pour être pratiqués. La Cour vise ensuite les pratiques croissantes consistant pour les demandeurs d'asile à déposer volontairement des photos ou des vidéos de leurs relations sexuelles comme modes de preuves. Dans la mesure où ces éléments de preuve mettent en scène l'intimité sexuelle de la personne, ils doivent être considérés comme dégradants. L'Avocate Générale avait alors précisé dans ses Conclusions que ces pratiques devaient rester interdites même en cas de consentement du demandeur ${ }^{51}$. En effet, comment ne pas avoir de sérieux doutes quant à la capacité d'un demandeur, en situation de grande vulnérabilité, de donner un consentement parfaitement libre et éclairé aux autorités nationales? Ce point n'a pas été précisé par la Cour et on peut le regretter. Toutefois, cela rejoint l'argument selon lequel autoriser de telles preuves emporterait "un effet incitatif à l'égard d'autres demandeurs et reviendrait, de facto, à imposer à ces derniers de telles preuves $»^{52}$. Si de telles preuves étaient acceptées, refuser de se soumettre à de telles pratiques risquerait d'être interprété négativement par les autorités nationales, c'est-à-dire comme le signe d'une faible crédibilité s. $^{3}$. Toute possibilité de donner un consentement libre et éclairé serait dès lors aléatoire et douteux.

36 A propos de l'ensemble de ces pratiques, la Cour précise, comme le défend d'ailleurs l'UNHCR ${ }^{54}$, que "de tels éléments n'ont pas nécessairement de valeur probante $»^{55}$. Cette position écho aux controverses scientifiques portant sur le caractère probant des tests de phallométrie. Elle renvoie encore aux propos de l'Avocate Générale pour qui de tels éléments de preuves (vidéos ou photographies) peuvent "être fabriqués» et ne pas permettre "de distinguer le demandeur véritable du faux demandeur $»^{56}$. Cette affirmation 
semble donc encore s'appuyer sur l'idée essentielle et sous-jacente selon laquelle l'orientation sexuelle d'un requérant ne s'appuie pas exclusivement sur son comportement sexuel. Comme l'explique l'UNHCR lui-même, il est en effet plus facile de se «fabriquer une expérience homosexuelle» que d'évoquer le processus complexe interne expliquant comment chaque personne vit son orientation sexuelle et son identité de genre ${ }^{57}$. Elle témoigne donc d'une compréhension large de l'orientation sexuelle.

De l'ensemble de ces motifs, il ressort que la production de tests, vidéos ou photographies sont strictement, et quelles que soient les circonstances, interdites même si certaines autorités nationales ont pu y voir un moyen d'objectiver les allégations du requérant. Seules les attestations médicales rédigées par des psychologues ou des psychiatres, qui font état que des traumatismes auxquels les requérants sont exposés en raison des persécutions qu'ils ont pu subir à cause de leur orientation sexuelle, restent autorisées.

\section{3 - L'illégalité des décisions de rejet se fondant exclusivement sur une révélation tardive de l'orientation sexuelle}

La Cour a affirmé de plus que retenir le défaut de crédibilité au seul motif que le requérant n'a pas invoqué son orientation sexuelle à la première occasion donnée n'est pas conforme à la Directive 2004/83/CE (Directive 2011/95/UE (refonte)) ${ }^{58}$. Si la Cour rappelle le droit des Etats de considérer qu'il appartient au demandeur de présenter " aussi rapidement que possible " tous les éléments nécessaires pour étayer sa demande, cette exigence est tempérée par l'article 13.3 a) et l'article 4.3 de cette même directive imposant aussi aux Etats de tenir compte de la situation personnelle et du statut individuel de chaque demandeur, et notamment sa vulnérabilité. De ce fait, en raison " du caractère sensible des questions ayant trait à la sphère personnelle", la Cour affirme qu'il ne saurait être conclu au défaut de crédibilité du seul fait de la réticence des demandeurs d'asile à "révéler des aspects intimes de sa vie ${ }^{59}$ ". Conforme à la position défendue par l'UNHCR ${ }^{60}$, cette position de la CJUE confirme que celle-ci fait droit à une conception non stéréotypée de l'orientation sexuelle. Elle s'appuie sur l'idée selon laquelle les capacités des personnes d'exposer des éléments intimes de leur identité peuvent être affectées selon les sentiments intériorisés de honte ou d'homophobie intériorisée, les traumatismes vécus, la difficulté de discerner toute l'ampleur des persécutions subies, l'âge, des facteurs culturels ou religieux propres à chacun.

Sans jamais entrer dans le débat d'une définition complexe de l'orientation sexuelle, la Cour montre à travers la fixation de ces trois limites infranchissables qu'elle ne la réduit pas à l'accomplissement d'actes sexuels mais la relie à l'identité de genre d'une personne. La Cour semble retenir une conception non stéréotypée de l'orientation sexuelle se fondant sur une approche individuelle et individualisée de tout examen prétendant vérifier la crédibilité de celle-ci. Toutefois, cette analyse est fortement ébranlée à la lecture du quatrième point de l'arrêt, à savoir celui portant sur l'appréciation de la légalité des questionnaires s'appuyant sur une conception volontairement stéréotypée de l'orientation sexuelle. 
4 - L'illégalité des décisions de rejet se fondant exclusivement sur des réponses dites « incorrectes » au regard d'une conception stéréotypée de l'orientation sexuelle

La Cour juge que l'incapacité des requérants de répondre "correctement " à des questionnaires fondés sur une conception stéréotypée des homosexuels ne peut pas être "un motif suffisant en vue de conclure au défaut de crédibilité" d'un demandeur d'asile, "en ce qu'il ne permet pas auxdites autorités de tenir compte de la situation individuelle et personnelle " des demandeurs d'asile ${ }^{61}$. A première vue, cela va notamment contraindre les autorités nationales à motiver leurs décisions de rejet sur des motifs qui ne pourront plus s'appuyer, comme c'est trop souvent le cas, sur une conception archétypale des homosexuels ${ }^{62}$. On comprend donc ici que de nombreuses décisions de rejet vont dès à présent pouvoir être contestées en justice. Toutefois, la Cour reconnaît explicitement que de tels types de questionnaires ne sont pas pour autant contraires à la Directive 2004/83/CE (Directive 2011/95/UE (refonte)). Elle va même jusqu'à expliquer qu'ils " peuvent constituer un élément utile à la disposition des autorités compétentes aux fins de cette évaluation ${ }^{63}$.

41 Autrement dit, la CJUE reconnaît que de tels questionnaires sont légaux et légitime. Pourquoi utiliser de tels questionnaires s'ils ne peuvent pas servir de fondement pour motiver une décision de reconnaissance ou non de la qualité de réfugié ? Pourquoi leur reconnaître une utilité si, dans le même temps, il est reconnu que la sexualité touche à l'expérience intime et identitaire de chaque individu ? La position de la Cour peut donc ici surprendre à la lumière des trois autres arguments développés plus haut, voire décevoir. Même si la Cour ne l'avait pas explicitement défendue, l'argumentation dégagée semblait au moins faire droit à l'affirmation d'une conception objective de l'orientation sexuelle. Mais, avec cette précision ajoutée, la Cour jette un doute sur cette interprétation et laisse ouvert le développement d'une approche stéréotypée de l'orientation sexuelle dans le cadre de l'examen des demandes d'asile. Plus concrètement, elle fixe aux Etats une voie à suivre extrêmement étroite voire complexe. Ils pourront continuer d'employer des questionnaires relevant d'une approche stéréotypée de l'orientation sexuelle, mais toute réponse "incorrecte » ne pourra pas fonder le motif d'une décision de rejet sauf à la justifier par l'identité individuelle de la personne.

Cet arrêt est à la fois fondamental dans les limites qu'il fixe et décevant dans l'argumentation qui les sous-tendent.

Il est fondamental car il met un terme définitif et général à l'emploi de méthodes abusives et choquantes pour apprécier la crédibilité d'une orientation sexuelle alléguée. La réponse de la Cour est précise pour les autorités nationales car les modes de preuves interdits sont identifiés ; elle est aussi suffisamment générale pour anticiper sur l'encadrement de toute nouvelle méthode intrusive que les agents pourraient mettre en place. 

conception non stéréotypée et non superficielle de l'orientation sexuelle. $\mathrm{Au}$ contraire, elle laisse ouvert la possibilité de recourir en toute légitimité à une telle conception pour élaborer les questionnaires servant à apprécier le caractère crédible d'une orientation sexuelle alléguée.

ll est encore décevant car la Cour a ignoré un pan entier des critères d'éligibilité au statut de réfugié, à savoir le critère des motifs imputés. Si les personnes craignant d'être persécutées en raison de leur orientation sexuelle peuvent se voir reconnaître la qualité de réfugié, tel est aussi le cas de celles qui, sans être homosexuelles, se voient imputées une orientation sexuelle différente et sont persécutées en raison de cette imputation. Par conséquent, toute la question est de savoir ce qui est "vraisemblablement» homosexuel n'a aucune importance. Ce qui compte est de savoir qui peut être considéré comme tel et donc être persécuté pour ce motif. Et, dans le cadre de cette question, se focaliser sur le comportement sexuel d'une personne est très insuffisant. C'est toute son identité personnelle, sexuelle et de genre, qu'il faut explorer pour apprécier si celle-ci s'oppose aux conceptions et rôles sociaux attribués à son sexe dans un pays.

CJUE, Grande Chambre, 2 décembre 2014, A, B \& $C$ c/ Staatssecretaris van Veiligheid en Justitie, Affaires jointes C-148/13, C-149/13 \& C-150/13.

Les Lettres « Actualités Droits-Libertés » (ADL) du CREDOF (pour s'y abonner) sont accessibles sur le site de la Revue des Droits de l'Homme (RevDH) - Contact

\section{NOTES}

1. CJUE, Quatrième Chambre, X., Y., \& Z. contre Minister voor Immigratie en Asiel, Affaires jointes C-199/12, C-200/12, C-201/12, 7 novembre 2013 - ADL du 13 novembre 2013.

2. Sabine JANSEN, Thomas SPIJKERBOER, «Fleeing Homophobia: Asylum Claims Related to Sexual Orientation and Gender Identity in Europe », Amsterdam, 2011, p. 47.

3. Par exemple, en Grande-Bretagne : Diane TAYLOR, Mark TOWSEND, « Gay asylum-seeker face 'Humiliation'", The Observer, 8 février 2014.

4. Eleanor V.E SHARPSTON, «Conclusions de l'avocat général présentées dans les affaires conjointes C-148/13, C-149/13 et C-150/13 », 17 juillet 2014, §28.

5. CJUE, Grande Chambre, Affaires jointes C-148/13, C-149/13 \& C-149/13, 2 décembre 2014, \$43.

6. CJUE, Quatrième Chambre, X., Y., \& Z. contre Minister voor Immigratie en Asiel, affaires jointes C-199/12, C-200/12, C-201/12, 7 novembre 2013.

7. Marie-Laure BASILIEN-GAINCHE, Caroline LANTERO, «Statut de réfugié et appartenance à un groupe social : Une victoire à la Pyrrhus pour les personnes homosexuelles », in Lettre "Actualités Droits-Libertés » du CREDOF, 13 novembre 2013. 
8. CJUE, Grande Chambre, Affaires jointes C-148/13, C-149/13 \& C-149/13, 2 décembre 2014, §52.

9. Marie-Laure BASILIEN- GAINCHE, «Droit de l'étranger à être entendu durant l'instruction d'une demande de protection subsidiaire ", in Lettres Actualités Droits-Libertés » du CREDOF, 24 novembre 2012.

10. CJUE, première Chambre, M.M. c/ Minister for Justice, Equality and Law Reform, Affaire C-277/11, du 22 novembre 2012, §64.

11. Ibid., $\$ 66$.

12. CJUE, première Chambre, M.M. c/ Minister for Justice, Equality and Law Reform, op. cit., $\$ 66$.

13. Mickaël KAGAN, «Is Truth in the Eye of the Beholder? Objective Credibility Assessment in Refugee Status Determination ", Georgetown Immigration Law Journal, n 367, 2003, p. 367-415 James S. SWEENEY, «Credibility, Proof and Refugee Law», International Journal of Refugee Law, vol. 21, n 4, 2009, p. 700-726; Jean-Michel BELORGEY, « Le contentieux du droit d'asile et l'intime conviction du juge ", Revue administrative, $\mathrm{n}^{\circ} 336$, novembre 2003, p. 619-622.

14. Marion TISSIER-RAFFIN, "La force probante des certificats médicaux dans l'appréciation du risque de violation de l'article 3 de la CEDH », Lettre «Actualités Droits-Libertés » du CREDOF, 23 octobre 2013.

15. Ce point est rappelé par Eleanor V. E SHARPSTON, op. cit., $\$ 65$.

16. Les principes de Jogjarkarta, Principes sur l'application de la législation internationale des droits humains en matière d'orientation sexuelle et d'identité de genre, mars 2007.

17. UNHCR, «Principes Directeurs sur la protection internationale $n^{\circ} 9$ : Demandes de statut de réfugié fondées sur l'orientation sexuelle et/ou l'identité de genre dans le contexte de l'article $1 \mathrm{~A}$ (2) de la Convention de 1951 et/ou de son protocole de 1967 relatifs au statut des réfugiés ", HCR/GIP/12/09, 23 octobre 2012.

18. Eleanor V.E SHARPSTON, op. cit., \$36; Les principes de Jogjarkarta, op. cit., §9.

19. UNHCR, « Principes Directeurs sur la protection internationale $n^{\circ} 9 . .$. », op. cit., $\$ 9,13 \& 63$.

20. CrEDH, $3^{\text {ème }}$ Sect, Van Kück c/ Allemagne, $n^{\circ}$ 35968/97, 12 septembre 2003.

21. UNHCR, « Principes Directeurs sur la protection internationale $n^{\circ} 9 . .$. », op. cit., $\$ 4 \& 63$.

22. CrEDH, $3^{\text {ème }}$ Sect., Van Kück c/ Allemagne, $n^{\circ} 35968 / 97,12$ septembre 2003, $\$ 69$.

23. Ces arguments sont repris dans Eleanor V.E SHARPSTON, "Conclusions de l'avocat général présentées dans les affaires conjointes C-148/13, C-149/13 et C-150/13», op. cit., \$37.

24. CJUE, Grande Chambre, Affaires jointes C-148/13, C-149/13 \& C-149/13, 2 décembre 2014, §49.

25. Ibid., §37.

26. Sabine JANSEN, Thomas SPIJKERBOER, op. cit., 93 p.

27. Colin YEO s'est exprimé dans la presse anglaise: Diane TAYLOR, Mark TOWSEND, «Gayasylum Seeker face 'Humiliation' ", The Observer, 8 février 2014.

28. Ibid..

29. John VINE CBE QPM, «An Investigation into the Home Office's Handling of Asylum Claims Made on the Grounds of Sexual Orientation ", Mars-Juin 2014, Londres: William Lea Group, 51 p. 30. Sabine JANSEN, Thomas SPIJKERBOER, op. cit., p. 54-57.

31. UNHCR, "UNHCR's Comments on the Practice of Phallometry in the Czech Republic to Determine the Credibility of Asylum Claims Based on Persecution due to Sexual Orientation ", UNHCR, Avril 2011.

32. Sabine JANSEN, Thomas SPIJKERBOER, op. cit., p. 49-53.

33. Ibid., p. 50-51.

34. CJUE, Grande Chambre, Affaires jointes C-148/13, C-149/13 \& C-149/13, 2 décembre 2014, $\S 23-24$.

35. Eleanor V. E SHARPSTON, op. cit., \$36 ; Les principes de Jogjarkarta, op. cit., \$22.

36. Eleanor V. E SHARPSTON, op. cit., \$36; Les principes de Jogjarkarta, op. cit., \$23.

37. CJUE, Grande Chambre, Affaires jointes C-148/13, C-149/13 \& C-149/13, 2 décembre 2014, \$26. 
38. Ibid., §28.

39. Eleanor V. E SHARPSTON, op. cit., \$36 ; Les principes de Jogjarkarta, op. cit., §24.

40. UNHCR, « Written Observations of the UNHCR - Joined Cases C-148/13, C-149/13 \& C-150/13 Minister Voor Immigratie en Asiel v. A, B \& C », UNHCR, 21 août 2013, § 3.3.

41. CJUE, Grande Chambre, Affaires jointes C-148/13, C-149/13 \& C-149/13, 2 décembre 2014, \$49.

42. Eleanor V. E SHARPSTON, op. cit., §48.

43. CJUE, Grande Chambre, Affaires jointes C-148/13, C-149/13 \& C-149/13, 2 décembre 2014, §56.

44. Ibid., §4.

45. Par exemple, à propos de requérants invoquant des persécutions religieuses, certaines cours nationales ont rappelé que les questions portant sur les connaissances de ces derniers d'une religion ne pouvaient attendre de réponses préconçues. En effet, la compréhension d'une religion peut varier selon des facteurs culturels et nationaux, et le statut des personnes (niveau d'éducation) : Wang c/ Canada, IMM-5187-11, 2012 CF 346, 22 mars 2012 ; Wang v. MIMA (2000) FCA 1599, 10 novembre 2000.

46. CJUE, Grande Chambre, Affaires jointes C-148/13, C-149/13 \& C-149/13, 2 décembre 2014, §46.

47. Ibid., §53.

48. UNHCR, "Written Observations of the UNHCR... », op. cit., §3. L’UNHCR a dressé une liste noire portant interdiction absolue de certaines pratiques et une liste grise portant interdiction d'autres pratiques selon leur manière d'être mises en œuvre.

49. CJUE, Grande Chambre, Affaires jointes C-148/13, C-149/13 \& C-149/13, 2 décembre 2014, \$64.

50. Ibid., $\$ 65$.

51. Eleanor V. E SHARPSTON, op. cit., \$66.

52. CJUE, Grande Chambre, Affaires jointes C-148/13, C-149/13 \& C-149/13, 2 décembre 2014, $\$ 66$.

53. UNHCR, « Written Observations of the UNHCR... », op. cit., \$3.13.

54. UNHCR, « Principes Directeurs sur la protection internationale $n^{\circ} 9 . . . »$, op. cit., $\$ 64$.

55. CJUE, Grande Chambre, Affaires jointes C-148/13, C-149/13 \& C-149/13, 2 décembre 2014, $\$ 65$.

56. Eleanor V. E SHARPSTON, op. cit., $\$ 66$.

57. UNHCR, « Written Observations of the UNHCR... », op. cit., \$3.9.

58. CJUE, Grande Chambre, Affaires jointes C-148/13, C-149/13 \& C-149/13, 2 décembre 2014, \$69-71.

59. CJUE, Grande Chambre, Affaires jointes C-148/13, C-149/13 \& C-149/13, 2 décembre 2014,\$69.

60. UNHCR, « Written Observations of the UNHCR... », op. cit., \$3.18.

61. CJUE, Grande Chambre, Affaires jointes C-148/13, C-149/13 \& C-149/13, 2 décembre 2014, $\$ 62$.

62. UNHCR, « Principes Directeurs sur la protection internationale $n^{\circ} 9 . . . »$, op. cit., $\$ 60$.

63. CJUE, Grande Chambre, Affaires jointes C-148/13, C-149/13 \& C-149/13, 2 décembre 2014, $\$ 62$.

\section{RÉSUMÉS}

Jusqu'à quel point une autorité compétente peut-elle aller lorsqu'elle vérifie les allégations d'homosexualité d'un demandeur d'asile pour déterminer s'il peut se voir reconnaitre la qualité de réfugié? Peut-elle interroger les demandeurs d'asile sur les détails de leurs pratiques sexuelles? Peut-elle accepter ou même exiger de ces derniers qu'ils apportent des photographies exposant leur vie sexuelle? Peut-elle aller jusqu'à s'appuyer sur des "tests pseudo-médicaux » en vue d'établir l'homosexualité du requérant? C'est sur ces pratiques, inégalement mises en œuvre dans plusieurs pays européens, que la CJUE était amenée à se 
prononcer dans l'arrêt C-148/13, C-149/13 et C-150/13 du 2 décembre 2014 à l'initiative d'une question préjudicielle une nouvelle fois posée par le Conseil d'état néerlandais. Se fondant sur la Directive 2004/83/ CE et la Charte des droits fondamentaux, la CJUE s'est clairement opposée aux dérives auxquelles la culture de la suspicion a pu mener certaines autorités dans leur examen de la crédibilité de l'orientation sexuelle invoquée par les demandeurs d'asile. Cet arrêt est de la plus haute importance pour les demandeurs d'asile invoquant des craintes de persécution en raison de leur orientation sexuelle. Il l'est aussi, de manière plus générale car les limites affirmées par la Cour, fondées sur le respect des droits fondamentaux des demandeurs d'asile et la Directive 2005/85/CE, ont une portée qui va au-delà de la seule appréciation de l'orientation sexuelle.

\section{AUTEUR}

\section{MARION TISSIER-RAFFIN}

Docteure en droit public (Université de Paris Ouest Nanterre La Défense - CREDOF) 